QCD Evolution Workshop 2014

International Journal of Modern Physics: Conference Series Vol. 37 (2015) 1560026 (8 pages)

(C) The Authors

DOI: $10.1142 / \mathrm{S} 2010194515600265$

\title{
Phenomenology of TMDs Using SCET
}

\author{
Stefano Melis* \\ Dipartimento di Fisica, Università di Torino, Via Pietro Giuria 1, \\ Torino, I-10125, Italy \\ stefano.melis@to.infn.it \\ Umberto D'Alesio \\ Dipartimento di Fisica, Università di Cagliari, Italy \\ INFN, Sezione di Cagliari, C. P. 170 \\ Monserrato (CA), I-09042, Italy \\ umberto.dalesio@ca.infn.it \\ Miguel G. Echevarria \\ Nikhef and Department of Physics and Astronomy, \\ VU University Amsterdam, De Boelelaan 1081, \\ HV Amsterdam, NL-1081, the Nerdelands \\ m.g.echevarria@nikhef.nl \\ Ignazio Scimemi \\ Departamento de Física Teórica II, \\ Universidad Complutense de Madrid (UCM), \\ Avenida Complutense s/n, Madrid, E-28040, Spain \\ ignazios@fis.ucm.es
}

Published 25 February 2015

\begin{abstract}
We present a global fit of current available data for Drell-Yan and $Z$-boson production using the Transverse Momentum Dependent evolution formalism of Refs. [1-4]. We performed our analysis including perturbative calculable contributions up to next-tonext-to-leading-logarithmic accuracy. We took into account non-perturbative corrections both to the intrinsic nucleon structure and to the evolution kernel.
\end{abstract}

Keywords: TMD evolution; resummation; Drell-Yan.

PACS numbers: 13.85.Qk, $12.38-\mathrm{t}$

*Speaker.

This is an Open Access article published by World Scientific Publishing Company. It is distributed under the terms of the Creative Commons Attribution 3.0 (CC-BY) License. Further distribution of this work is permitted, provided the original work is properly cited. 


\section{Introduction and formalism}

In this contribution we present a study of Drell-Yan (DY) and Z-boson production data in the context of the Transverse Momentum Dependent (TMD) evolution scheme developed in Refs. [1-4]. DY and $Z$-production processes are particularly useful in order to study TMD evolution. In fact, in these processes only TMD Parton Distribution Functions (TMDPDFs) are involved, contrary to other processes like the Semi-Inclusive Deep Inelastic Scattering (SIDIS) where the TMDPDFs appear convoluted with the TMD fragmentation functions. Moreover, present DY/Z data span a wide region in the mass of the virtual boson, $Q$, and in its transverse momentum, $q_{T}$, thus, allowing the study of the TMDPDFs in both perturbative and non-perturbative regimes.

By means of the factorization theorem, we can write the DY cross section as:

$$
\begin{aligned}
\frac{d \sigma}{\sigma_{0} d Q^{2} d q_{T}^{2} d y}= & H\left(Q^{2}, \mu^{2}\right) \sum_{q} e_{q}^{2} \int \frac{d^{2} \boldsymbol{b}_{T}}{4 \pi} e^{-i \boldsymbol{q}_{T} \cdot \boldsymbol{b}_{T}} \times \\
& \left\{\tilde{F}_{q / A}\left(x_{A}, b_{T} ; \zeta_{A}, \mu\right) \tilde{F}_{\bar{q} / B}\left(x_{B}, b_{T} ; \zeta_{B}, \mu\right)\right\}+Y,
\end{aligned}
$$

where:

$$
\sigma_{0}=\frac{4 \pi \alpha_{\mathrm{em}}^{2}}{9 Q^{2} s}, \quad x_{A, B}=\sqrt{\tau} e^{ \pm y}, \quad \tau=\frac{Q^{2}+q_{T}^{2}}{s} \simeq \frac{Q^{2}}{s} .
$$

Here, $\tilde{F}_{q / N}\left(x, b_{T} ; \zeta, \mu\right)$ is the TMDPDF in the $b_{T}$ space for a parton $q$ in a hadron $N$; $\mu$ is the factorization scale while $\zeta$ is a scale related to the the rapidity divergences that appear in the definition of the TMD soft functions, see Ref. [1] for more details; $H\left(Q^{2}, \mu^{2}\right)$ is a process dependent hard factor; $Y$ is the part of the collinear cross section (computed at fixed order in $\alpha_{s}$ ) which is regular at $q_{T} \rightarrow 0$.

Since the TMDPDF contains a non-perturbative part which is relevant at low energy and that must be modeled, it is useful to evolve the TMDPDF from a low initial scale $\left(\mu_{i}, \zeta_{i}\right)$, where we can easily set a model, up to an higher scale $\left(\mu_{f}, \zeta_{f}\right)$. This can be done by means of an evolutor. In impact parameter space we have that

$$
\tilde{F}\left(x, b_{T} ; \zeta_{f}, \mu_{f}\right)=\tilde{R}\left(b_{T} ; \zeta_{i}, \mu_{i}, \zeta_{f}, \mu_{f}\right) \tilde{F}\left(x, b_{T} ; \zeta_{i}, \mu_{i}\right),
$$

where $\tilde{R}$ is the evolutor. The expression for the evolutor is given by:

$$
\tilde{R}\left(b_{T} ; \zeta_{i}, \mu_{i}, \zeta_{f}, \mu_{f}\right)=\exp \left\{\int_{\mu_{i}}^{\mu_{f}} \frac{d \bar{\mu}}{\bar{\mu}} \gamma_{F}\left(\alpha_{s}(\bar{\mu}), \ln \frac{\zeta_{f}}{\bar{\mu}^{2}}\right)\right\}\left(\frac{\zeta_{f}}{\zeta_{i}}\right)^{-D\left(b_{T} ; \mu_{i}\right)}
$$

where $\gamma_{F}$ is the anomalous dimension of the TMDPDF and is related to the anomalous dimension of the hard factor as explained in Ref. [1]. The function $D$ follows the renormalization group equation

$$
\frac{d D}{d \ln \mu}=\Gamma_{\text {cusp }}
$$


where $\Gamma_{\text {cusp }}$ is the cusp anomalous dimension. Solving this equation with respect to $\mu_{b}=2 e^{-\gamma_{E}} / b_{T}$ one gets:

$$
D\left(b_{T} ; \mu\right)=D\left(b_{T} ; \mu_{b}\right)+\int_{\alpha_{s}\left(\mu_{b}\right)}^{\alpha_{s}(\mu)} d \alpha^{\prime} \frac{\Gamma_{\mathrm{cusp}}\left(\alpha^{\prime}\right)}{\beta\left(\alpha^{\prime}\right)},
$$

where $\beta\left(\alpha_{s}\right)$ is the QCD $\beta$-function. Writing $\alpha_{s}\left(\mu_{b}\right)$ as a series in $\alpha_{s}(\mu)$, one can resum $^{3}$ all the logs of the type $L_{T}=\ln \left(\mu^{2} / \mu_{b}^{2}\right)$, obtaining a resummed $D^{R}$ function:

$$
\begin{aligned}
D^{R}\left(b_{T} ; \mu\right)= & -\frac{\Gamma_{0}}{2 \beta_{0}} \ln (1-X) \\
& +\frac{1}{2}\left(\frac{a_{s}}{1-X}\right)\left[-\frac{\beta_{1} \Gamma_{0}}{\beta_{0}^{2}}(X+\ln (1-X))+\frac{\Gamma_{1}}{\beta_{0}} X\right] \\
& +\frac{1}{2}\left(\frac{a_{s}}{1-X}\right)^{2}\left[2 d_{2}(0)+\frac{\Gamma_{2}}{2 \beta_{0}}(X(2-X))\right. \\
& +\frac{\beta_{1} \Gamma_{1}}{2 \beta_{0}^{2}}(X(X-2)-2 \ln (1-X)) \\
& \left.+\frac{\beta_{2} \Gamma_{0}}{2 \beta_{0}^{2}} X^{2}+\frac{\beta_{1}^{2} \Gamma_{0}}{2 \beta_{0}^{3}}\left(\ln ^{2}(1-X)-X^{2}\right)\right],
\end{aligned}
$$

where we have used the notation

$$
a_{s}=\frac{\alpha_{s}(\mu)}{4 \pi}, \quad X=a_{s} \beta_{0} L_{T}, \quad L_{T}=\ln \frac{\mu^{2} b_{T}^{2}}{4 e^{-2 \gamma_{E}}}=\ln \frac{\mu^{2}}{\mu_{b}^{2}} .
$$

This resummation is valid only in a perturbative regime therefore we can write:

$$
\tilde{R}^{\text {pert }}\left(b_{T} ; \zeta_{i}, \mu_{i}, \zeta_{f}, \mu_{f}\right)=\exp \left\{\int_{\mu_{i}}^{\mu_{f}} \frac{d \bar{\mu}}{\bar{\mu}} \gamma_{F}\left(\alpha_{s}(\bar{\mu}), \ln \frac{\zeta_{f}}{\bar{\mu}^{2}}\right)\right\}\left(\frac{\zeta_{f}}{\zeta_{i}}\right)^{-D^{R}\left(b_{T} ; \mu_{i}\right)}
$$

The complete form of the evolution kernel contains a non-perturbative part; we then write:

$$
\tilde{R}\left(b_{T} ; \zeta_{i}, \mu_{i}, \zeta_{f}, \mu_{f}\right)=\tilde{R}^{\text {pert }}\left(b_{T} ; \zeta_{i}, \mu_{i}, \zeta_{f}, \mu_{f}\right)\left(\frac{\zeta_{f}}{\zeta_{i}}\right)^{-D^{\mathrm{NP}}\left(b_{T}\right)}
$$

where $D^{\mathrm{NP}}$ is the non-perturbative piece of the $D$ function. Notice that the relevance of $D^{\mathrm{NP}}$ can not be predicted a priori and only the experimental data can reveal its role.

In order to make a study of the unpolarized TMDPDF using Eq. (3) we need a model at the initial scale. The main constraint on the structure of TMDPDFs comes from their limit at high transverse momentum, $q_{T} \gg \Lambda_{\mathrm{QCD}}$. In this case, the 
TMDPDFs can be written as the sum of convolutions of Wilson coefficients and collinear Parton Distribution Functions (PDFs): ${ }^{1,5}$

$$
\tilde{F}_{q / N}\left(x, b_{T} ; \zeta, \mu\right)=\left(\frac{\zeta}{\mu_{b}^{2}}\right)^{-D\left(b_{T} ; \mu\right)} \sum_{j} \int_{x}^{1} \frac{d z}{z}\left\{\tilde{C}_{q \leftarrow j}^{\phi}\left(\frac{x}{z}, b_{T} ; \mu\right) f_{j / N}(z ; \mu)\right\} .
$$

The Wilson coefficients $\tilde{C}^{\Phi}$ for the quark-quark and the quark-gluon channel at one loop are: ${ }^{1}$

$$
\begin{aligned}
\tilde{C}_{q \leftarrow q^{\prime}}^{\mathscr{Q}}\left(z, b_{T} ; \mu\right)= & \delta(1-z) \delta_{q q^{\prime}}+2 a_{s} C_{F}\left[1-z-\mathcal{P}_{q \leftarrow q^{\prime}} L_{T}\right. \\
& \left.-\delta(1-z)\left(-\frac{1}{2} L_{T}^{2}-\frac{3}{2} L_{T}+\frac{\pi^{2}}{12}\right)\right] \delta_{q q^{\prime}}, \\
\tilde{C}_{q \leftarrow g}^{\phi}\left(z, b_{T} ; \mu\right)= & 2 a_{s} T_{F}\left[2 z(1-z)-\mathcal{P}_{q \leftarrow g} L_{T}\right]
\end{aligned}
$$

and the one-loop splitting kernels appearing above are given by

$$
\mathcal{P}_{q \leftarrow q}=\left(\frac{1+z^{2}}{1-z}\right)_{+}, \quad \mathcal{P}_{q \leftarrow g}=z^{2}+(1-z)^{2} .
$$

Under renormalization group equation one has:

$$
\begin{aligned}
\frac{d}{d \ln \mu} \tilde{C}_{q \longleftarrow j}^{\phi}\left(z, b_{T} ; \mu\right)= & \left(\Gamma_{\text {cusp }} L_{T}-\gamma^{V}\right) \tilde{C}_{q \leftarrow j}^{\Phi}\left(z, b_{T} ; \mu\right) \\
& -\sum_{i} \int_{z}^{1} \frac{d \xi}{\xi} \tilde{C}_{q \leftarrow i}^{\Phi}\left(\xi, b_{T} ; \mu\right) \mathcal{P}_{i \leftarrow j}(z / \xi) .
\end{aligned}
$$

An explicit expression of $\gamma^{V}$ can be found in Ref. [1]. The double logarithms in Eq. (15) can be partially exponentiated ${ }^{6,7}$ obtaining:

$$
\tilde{C}_{q \leftarrow j}^{\Phi}\left(z, b_{T} ; \mu\right) \equiv \exp \left[h_{\Gamma}\left(b_{T} ; \mu\right)-h_{\gamma}\left(b_{T} ; \mu\right)\right] \hat{C}_{q \leftarrow j}\left(z, b_{T} ; \mu\right),
$$

where $h_{\Gamma}$ and $h_{\gamma}$ are defined by the following renormalization equations:

$$
\frac{d h_{\Gamma}}{d \ln \mu}=\Gamma_{\mathrm{cusp}} L_{T} ; \quad \frac{d h_{\gamma}}{d \ln \mu}=\gamma^{V} .
$$

$\Gamma_{\text {cusp }}, \gamma^{V}$ and $h_{\Gamma, \gamma}$ can be perturbatively expanded in series of $\alpha_{s}$, see Refs. [3, 4] for their explicit expressions. We can solve the evolution equation for $h_{\Gamma, \gamma}$ obtaining:

$$
\begin{aligned}
& h_{\Gamma}\left(b_{T} ; \mu\right)=h_{\Gamma}\left(b_{T} ; \mu_{b}\right)+\int_{\mu_{b}}^{\mu} \frac{d \bar{\mu}}{\bar{\mu}} \Gamma_{\text {cusp }} L_{T}, \\
& h_{\gamma}\left(b_{T} ; \mu\right)=h_{\gamma}\left(b_{T} ; \mu_{b}\right)+\int_{\mu_{b}}^{\mu} \frac{d \bar{\mu}}{\bar{\mu}} \gamma^{V} .
\end{aligned}
$$

The expressions above still contain large logarithms $L_{T}$ that need to be resummed when $\alpha_{s} L_{T}$ is of order 1 . We can apply the method of integration used for the 
evaluation of $D^{R}$ to find $h_{\gamma}^{R}$ as the equation for $h_{\gamma}$ (second line of Eq. (18)) has the same functional form as Eq. (6), so that

$$
\begin{aligned}
h_{\gamma}^{R}\left(b_{T} ; \mu\right)=-\frac{\gamma_{0}}{2 \beta_{0}} \ln (1-X) & +\frac{1}{2}\left(\frac{a_{s}}{1-X}\right)\left[-\frac{\beta_{1} \gamma_{0}}{\beta_{0}^{2}}(X+\ln (1-X))+\frac{\gamma_{1}}{\beta_{0}} X\right] \\
+\frac{1}{2}\left(\frac{a_{s}}{1-X}\right)^{2}[ & \frac{\gamma_{2}}{2 \beta_{0}}(X(2-X))+\frac{\beta_{2} \gamma_{0}}{2 \beta_{0}^{2}} X^{2} \\
& +\frac{\beta_{1} \gamma_{1}}{2 \beta_{0}^{2}}(X(X-2)-2 \ln (1-X)) \\
& \left.+\frac{\beta_{1}^{2} \gamma_{0}}{2 \beta_{0}^{3}}\left(\ln ^{2}(1-X)-X^{2}\right)\right] .
\end{aligned}
$$

In the case of $h_{\Gamma}$ we note that $L_{T}=\int_{\alpha_{s}\left(\mu_{b}\right)}^{\alpha_{s}(\mu)} \frac{d \alpha}{\beta(\alpha)}$, and in this way Eq. (18) (first line) can be solved as

$$
h_{\Gamma}^{R}\left(b_{T} ; \mu\right)=\int_{\alpha_{s}\left(\mu_{b}\right)}^{\alpha_{s}(\mu)} d \alpha^{\prime} \frac{\Gamma_{\text {cusp }}\left(\alpha^{\prime}\right)}{\beta\left(\alpha^{\prime}\right)} \int_{\alpha_{s}\left(\mu_{b}\right)}^{\alpha^{\prime}} \frac{d \alpha}{\beta(\alpha)} .
$$

One can perform the above integration first expanding the $\beta$-function and finally re-writing $\alpha_{s}\left(\mu_{b}\right)$ in terms of $\alpha_{s}(\mu)$ at the correct order, as shown in Ref. [3]. The result is:

$$
\begin{aligned}
h_{\Gamma}^{R}\left(b_{T} ; \mu\right)= & \frac{\Gamma_{0}(X-(X-1) \ln (1-X))}{2 a_{s} \beta_{0}^{2}} \\
& +\frac{\beta_{1} \Gamma_{0}\left(2 X+\ln ^{2}(1-X)+2 \ln (1-X)\right)-2 \beta_{0} \Gamma_{1}(X+\ln (1-X))}{4 \beta_{0}^{3}} \\
& +\frac{a_{s}}{4 \beta_{0}^{4}(1-X)}\left(\beta_{0}^{2} \Gamma_{2} X^{2}-\beta_{0}\left(\beta_{1} \Gamma_{1}(X(X+2)+2 \ln (1-X))\right.\right. \\
& \left.+\beta_{2} \Gamma_{0}((X-2) X+2(X-1) \ln (1-X))\right) \\
& \left.+\beta_{1}^{2} \Gamma_{0}(X+\ln (1-X))^{2}\right),
\end{aligned}
$$

while for the $\hat{C}$ 's one gets:

$$
\begin{aligned}
& \hat{C}_{q \leftarrow q^{\prime}}\left(z, b_{T} ; \mu\right)=\left\{\delta(1-z)+2 a_{s} C_{F}\left[1-z-\delta(1-z) \frac{\pi^{2}}{12}-\mathcal{P}_{q \leftarrow q^{\prime}} L_{T}\right]\right\} \delta_{q q^{\prime}} \\
& \hat{C}_{q \leftarrow g}\left(z, b_{T} ; \mu\right)=2 a_{s} T_{F}\left[2 z(1-z)-\mathcal{P}_{q \leftarrow g} L_{T}\right] .
\end{aligned}
$$

Thus, the fully resummed perturbative part of the TMDPDF reads:

$$
\begin{aligned}
\tilde{F}_{q / N}^{\text {pert }}\left(x, b_{T} ; \zeta, \mu\right)= & \left(\frac{\zeta}{\mu_{b}}\right)^{-D^{R}\left(b_{T} ; \mu\right)} e^{h_{\Gamma}^{R}\left(b_{T} ; \mu\right)-h_{\gamma}^{R}\left(b_{T} ; \mu\right)} \\
& \sum_{j} \int_{x}^{1} \frac{d z}{z} \hat{C}_{q \leftarrow j}\left(x / z, b_{T} ; \mu\right) f_{j / N}(z ; \mu) .
\end{aligned}
$$




\section{Phenomenological analysis}

Eq. (23) has been obtained resumming perturbatively the large logs. Therefore, it is valid only in the perturbative region. The large $-b_{T}$ region of the TMDPDF must be corrected for non-perturbative contributions:

$$
\tilde{F}_{q / N}\left(x, b_{T} ; Q_{i}^{2}, \mu_{i}\right)=\tilde{F}_{q / N}^{\text {pert }}\left(x, b_{T} ; Q_{i}^{2}, \mu_{i}\right) \tilde{F}_{q / N}^{\mathrm{NP}}\left(x, b_{T}\right)\left(\frac{Q_{i}^{2}}{Q_{0}^{2}}\right)^{-D^{\mathrm{NP}}\left(b_{T}\right)},
$$

where we set $\zeta \equiv Q^{2}$ and $\tilde{F}_{q / N}^{\mathrm{NP}}\left(x, b_{T}\right)$ is the non-perturbative part of the TMDPDF.

We notice that the splitting into a coefficient and a PDF is valid only at high transverse momentum. In order to minimize the logarithms generated by this splitting we choose $\mu_{i} \equiv \sqrt{\zeta_{i}} \equiv Q_{i}=Q_{0}+q_{T}$ where $Q_{0}$ is a fixed low scale. In our analysis we choose $Q_{0} \sim 2 \mathrm{GeV}$.

Collecting all results in Eqs. (3), (10) and (24) we can write the resummed TMDPDF that enters into the factorization theorem as

$$
\begin{aligned}
\tilde{F}_{q / N}\left(x, b_{T} ; Q^{2}, Q\right)= & \exp \left\{\int_{Q_{i}}^{Q} \frac{d \bar{\mu}}{\bar{\mu}} \gamma_{F}\left(\alpha_{s}(\bar{\mu}), \ln \frac{Q^{2}}{\bar{\mu}^{2}}\right)\right\} \\
& \times\left(\frac{Q^{2}}{\mu_{b}^{2}}\right)^{-D^{R}\left(b_{T} ; Q_{i}\right)} e^{h_{\Gamma}^{R}\left(b_{T} ; Q_{i}\right)-h_{\gamma}^{R}\left(b_{T} ; Q_{i}\right)} \\
& \times \sum_{j} \int_{x}^{1} \frac{d z}{z} \hat{C}_{q \leftarrow j}\left(x / z, b_{T} ; Q_{i}\right) f_{j / N}\left(z ; Q_{i}\right) \\
& \times \tilde{F}_{q / N}^{\mathrm{NP}}\left(x, b_{T}\right)\left(\frac{Q_{i}^{2}}{Q_{0}^{2}}\right)^{-D^{\mathrm{NP}}\left(b_{T}\right)},
\end{aligned}
$$

where we set $\mu_{f} \equiv \sqrt{\zeta_{F}} \equiv Q$ and $\mu_{i} \equiv \sqrt{\zeta_{i}} \equiv Q_{i}=Q_{0}+q_{T}$.

In our analysis we considered the low energy Drell-Yan data of the Fermilab E288 ${ }^{8}$ experiment at three different energies of the beam $(200,300,400 \mathrm{GeV} / c)$, the CERN R209 data, ${ }^{9}$ and the Tevatron data of CDF Run I [10] and Run II [11] and D0 Run I $[12,13]$ and Run II [14]. We performed the analysis at Next-to-LeadingLogarithmic (NLL) and NNLL accuracy. Correspondingly, for the collinear PDF we used the MSTW08 PDF set ${ }^{15}$ at Next Leading Order (NLO) and Next to NLO (NNLO). Assuming $D^{\mathrm{NP}}=0$ in Eq. (25), we found that data can be described by the following parametrization of the non-perturbative function $\tilde{F}^{\mathrm{NP}}$ :

$$
\tilde{F}_{q / N}^{\mathrm{NP}}\left(x, b_{T}\right)=e^{-\lambda_{1} b_{T}}\left(1+\lambda_{2} b_{T}^{2}\right),
$$

where $\lambda_{1}$ and $\lambda_{2}$ are free parameters. Because CERN R209 and Fermilab E288 data are affected by large uncertainties in the normalization of the cross section we allow for two extra parameters in the fit, namely the normalization factors for E288 and R209 data: $N_{\mathrm{E} 288}$ and $N_{\mathrm{R} 209}$. Therefore, we have, in total, four free parameters: $\lambda_{1}, \lambda_{2}, N_{\mathrm{E} 288}$ and $N_{\mathrm{R} 209}$. The results of our fits are summarized in Tables (1) and (2). We found that both NLL-NLO and NNLL-NNLO calculations 
Table 1. Results of our global fit on low-energy and Tevatron data, with $D^{\mathrm{NP}}=0$ (Eq. (26)), $Q_{i}=Q_{0}+q_{T}$, at NNLL and NNL accuracies and with the collinear parton distributions from MSTW08 at NNLO and NLO.

\begin{tabular}{lll}
\hline NLL & 223 points & $\chi^{2} /$ d.o.f. $=1.51$ \\
\hline & $\lambda_{1}=0.26_{-0.02_{\text {th }}^{+0.0}}^{+0.05_{\text {th }}} \pm 0.05_{\text {stat }} \mathrm{GeV}$ & $\lambda_{2}=0.13 \pm 0.01_{\text {th }} \pm 0.03_{\text {stat }} \mathrm{GeV}^{2}$ \\
& $N_{\mathrm{E} 288}=0.9_{-0.1_{\text {th }}}^{+0.004_{\text {stat }}}$ & $N_{\text {R209 }}=1.3 \pm 0.01_{\text {th }} \pm 0.2_{\text {stat }}$ \\
\hline NNLL & 223 points & $\chi^{2} /$ d.o.f. $=1.12$ \\
\hline & $\lambda_{1}=0.33 \pm 0.02_{\text {th }} \pm 0.05_{\text {stat }} \mathrm{GeV}$ & $\lambda_{2}=0.13 \pm 0.01_{\text {th }} \pm 0.03_{\text {stat }} \mathrm{GeV}^{2}$ \\
& $N_{\text {E288 }}=0.85 \pm 0.01_{\text {th }} \pm 0.04_{\text {stat }}$ & $N_{\text {R209 }}=1.5 \pm 0.01_{\text {th }} \pm 0.2_{\text {stat }}$ \\
\hline
\end{tabular}

Table 2. Total and partial $\chi^{2} /$ points of our global fit on low-energy and Tevatron data with $D^{\mathrm{NP}}=0$ (Eq. (26)), $Q_{i}=Q_{0}+q_{T}$, at NNLL and NNL accuracies and with the collinear parton distributions from MSTW08 at NNLO and NLO.

\begin{tabular}{cccc}
\hline & & NNLL, NNLO & NLL, NLO \\
\hline & points & $\chi^{2} /$ points & $\chi^{2} /$ points \\
\hline & 223 & 1.10 & 1.48 \\
E288 200 & 35 & 1.53 & 2.60 \\
E288 300 & 35 & 1.50 & 1.12 \\
E288 400 & 49 & 2.07 & 1.79 \\
R209 & 6 & 0.16 & 0.25 \\
CDF Run I & 32 & 0.74 & 1.31 \\
D0 Run I & 16 & 0.43 & 1.44 \\
CDF Run II & 41 & 0.30 & 0.62 \\
D0 Run II & 9 & 0.61 & 2.40 \\
\hline
\end{tabular}

give a good description of the data. In particular NNLL-NNLO calculation gives a better total $\chi^{2}$, with a better description of Tevatron data. Notice that high-energy Tevatron data correspond to a region where $Q \equiv M_{Z}$ and $q_{T}$ spans a region from one up to tens of GeVs. This means that, in the Fourier impact parameter space, we are exploring the small $b_{T}$ region, that is, the region where the (perturbative) resummation of large logarithms plays an important role. Comparing the first and the second column of Tab. (2) we can see that, as expected, the description of Tevatron data improves going from NLL to NNLL accuracy. In Ref. [4] we explored the role played by the collinear PDFs adopting another set, namely the CTEQ10 [16] and we find a complete consistency among the results. We also checked the role played by a possible $D^{\mathrm{NP}}$ function different from zero. We did not find a conclusive evidence of such a term in present DY/Z data. However this term may be relevant for very low energy data sets like the SIDIS data sets of the HERMES and COMPASS experiments. 


\section{Acknowledgments}

M.G.E. is supported by the "Stichting voor Fundamenteel Onderzoek der Materie" (FOM), which is financially supported by the "Nederlandse Organisatie voor Wetenschappelijk Onderzoek" (NWO). U.D. and S.M. acknowledges support from the European Community under the FP7 program "Capacities - Research Infrastructures" (HadronPhysics3, Grant Agreement 283286). S.M. is partly supported by the "Progetto di Ricerca Ateneo/CSP" (codice TO-Call3-2012-0103). I.S. is supported by the Spanish MECD grant, FPA2011-27853-CO2-02.

\section{References}

1. M. G. Echevarria, A. Idilbi and I. Scimemi, JHEP 1207, 002 (2012).

2. M. G. Echevarra, A. Idilbi and I. Scimemi, Phys. Lett. B 726, 795 (2013).

3. M. G. Echevarria, A. Idilbi, A. Schfer and I. Scimemi, Eur. Phys. J. C 73, 2636 (2013).

4. U. D'Alesio, M. G. Echevarria, S. Melis and I. Scimemi, arXiv:1407.3311 [hep-ph] (2014).

5. J. Collins, Foundations of perturbative QCD (Cambridge monographs on particle physics, nuclear physics and cosmology. 32), (2011).

6. J. Kodaira and L. Trentadue, Phys. Lett. B 112, 66 (1982).

7. T. Becher and M. Neubert, Eur. Phys. J. C 71, 1665 (2011).

8. A. S. Ito, R. J. Fisk, H. Jostlein, D. M. Kaplan, S. W. Herb, D. C. Hom, L. M. Lederman and H. D. Snyder et al., Phys. Rev. D 23, 604 (1981).

9. D. Antreasyan, W. Atwood, U. Becker, G. Bellettini, P. L. Braccini, J. G. Branson, J. D. Burger and F. Carbonara et al., Phys. Rev. Lett. 47, 12 (1981).

10. T. Affolder et al. [CDF Collaboration], Phys. Rev. Lett. 84, 845 (2000).

11. T. Aaltonen et al. [CDF Collaboration], Phys. Rev. D 86, 052010 (2012).

12. B. Abbott et al. [D0 Collaboration], Phys. Rev. Lett. 84, 2792 (2000).

13. B. Abbott et al. [D0 Collaboration], Phys. Rev. D 61, 032004 (2000).

14. V. M. Abazov et al. [D0 Collaboration], Phys. Rev. Lett. 100, 102002 (2008).

15. A. D. Martin, W. J. Stirling, R. S. Thorne and G. Watt, Eur. Phys. J. C 63, 189 (2009).

16. H.-L. Lai, M. Guzzi, J. Huston, Z. Li, P. M. Nadolsky, J. Pumplin and C. -P. Yuan, Phys. Rev. D 82, 074024 (2010). 